\title{
High-resolution photoemission from a tunable quantum well: $\mathrm{Cu}(111) / \mathrm{Na}$
}

\author{
A. Carlsson, B. Hellsing, S.- $\AA$. Lindgren, and L. Walldén \\ Physics Department, Chalmers University of Technology, 41296 Göteborg, Sweden
}

(Received 23 December 1996)

\begin{abstract}
High-resolution photoemission $(\Delta E=5 \mathrm{meV})$ at a low photon energy $(3.82 \mathrm{eV})$ is used to probe discrete quantum-well-type subband states near, below, and above $E_{F}$ for $\mathrm{Cu}(111)$ covered with $2 \mathrm{ML}$ or less of $\mathrm{Na}$. A subband characteristic of the monolayer range shifts gradually to lower energy as the coverage is increased, extending below the Fermi level for coverages above 0.85 ML. Combined with previous observations of shifts for filled and empty states the present results show that the Na monolayer has continuously tunable quantumwell state energies. Beyond the monolayer range quantum-well states characteristic of both one and two atomic layers are observed, indicating growth of the second layer via monolayer high islands. A small downshift, by $25 \mathrm{meV}$, with increasing coverage in the second layer is ascribed to an increase of the island size. Lorentzian photoemission line shapes are observed for well-ordered samples. The linewidth varies linearly with temperature in the probed range (130-295 K) and this is ascribed to the phonon contribution to the width. Structural disorder leads to an asymmetric line, which is Lorentzian on the steeper, low-kinetic-energy side. [S0163-1829(97)00627-9]
\end{abstract}

\section{INTRODUCTION}

Alkali-metal monolayers on metal substrates have long attracted interest as examples of simple chemisorption systems ${ }^{1}$ and, more recently, as examples of simple metal quantum wells. ${ }^{2}$ One main objective of electron spectroscopic work is to monitor the development, as more alkali metal is adsorbed, of the overlayer into a slab of metal with properties different from those of the substrate when this is either clean or covered with an array of dispersed alkalimetal atoms. Some of the recent work has focused on the coverage dependence of the valence electron structure as the alkali metal is adsorbed on a metal substrate, such as $\mathrm{Cu}(111)$ (Ref. 3) and $\mathrm{Be}(0001)$ (Ref. 4), with, for a certain range of $k_{\|}$values, a band gap about the Fermi level. In these cases discrete quantum-well-type states may form in the overlayer and be detected by photoemission or inverse photoemission methods. One question of interest is whether the electronic structure depends on coverage in a gradual manner or if, as suggested, ${ }^{3,4}$ there is at some coverage a stepwise change associated with a structural transformation occurring maybe in concert with an onset of metal character.

Here we report on angle-resolved photoelectron spectra recorded at a low photon energy $(3.82 \mathrm{eV})$ and with a high energy resolution $(5 \mathrm{meV})$ for $\mathrm{Cu}(111)$ covered with between 0.7 and 2 atomic layers of $\mathrm{Na}$. The light source is a 1-mW He-Cd laser that gives sufficient emission intensity to observe emission out of rather weakly populated states in an energy range extending to around $10 \mathrm{kT}$ above the Fermi level. This is an important energy range where the present photoemission results provide a useful complement to electron spectroscopic results for states above $E_{F}$, obtained by inverse photoemission (IPES) and two-photon photoemission (PPES). For alkali monolayers the energy range near $E_{F}$ is of particular interest for observing states responsible for the development of an overlayer with metal character.

The overlayer states observed for 1 and $2 \mathrm{ML}$ of $\mathrm{Na}$ on $\mathrm{Cu}(111)$ have energies within $0.1 \mathrm{eV}$ of $E_{F}$. This means that the photoemission lines are narrow, having widths that are due mainly to thermal vibrations and static disorder. For the monolayer state both of these sources of broadening are observed. In the temperature range studied, 130-295 K, the thermal vibrations give Lorentzian lines with an energy spread proportional to $T$. A marked asymmetry of emission peak is observed at submonolayer coverages and this is ascribed to the disordered structure of the overlayer. Some of the results discussed below have been presented in previously. $^{5}$

\section{EXPERIMENT}

The photoelectron energy distributions are recorded with a spectrometer used also for high-resolution electron-energyloss measurements (Leybold EL22). At the settings used for the present work the elastic peak has a width of $7 \mathrm{meV}$. The emission from the overlayer states is due to $p$-polarized light. The intensity of $p$-polarized light may be varied via a $\lambda / 4$ plate or damping filters inserted in the beam. A reduced intensity is necessary at some coverages. At high monolayer coverages light from the $1-\mathrm{mW} \mathrm{He}-\mathrm{Cd}$ laser $(h \nu=3.82 \mathrm{eV}$, incident at an angle of $79^{\circ}$ ) would produce a count rate exceeding $10^{6} \mathrm{~s}^{-1}$ and saturate the detector. $\mathrm{Na}$ is evaporated at a rate of around $10 \mathrm{~min}$ per atomic layer onto a $\mathrm{Cu}(111)$ crystal cleaned by argon ion bombardment $(1 \mu \mathrm{A}, 500 \mathrm{eV})$ followed by annealing $(600 \mathrm{~K})$. $\mathrm{Na}$ is evaporated from a glass ampoule broken in situ and held at constant temperature during an experimental run.

The temperature at the sample surface is measured via the width of the Fermi edge. The adsorbed amount of alkali metal is obtained from the deposition time using as a reference the evaporation time needed to produce one full monolayer. At this coverage the emission from the monolayer state has nearly maximum intensity and the energy shift of a state characteristic of the monolayer saturates. As discussed further below there is a small difference $(4 \%)$ between the evaporation times needed to give maximum intensity and 


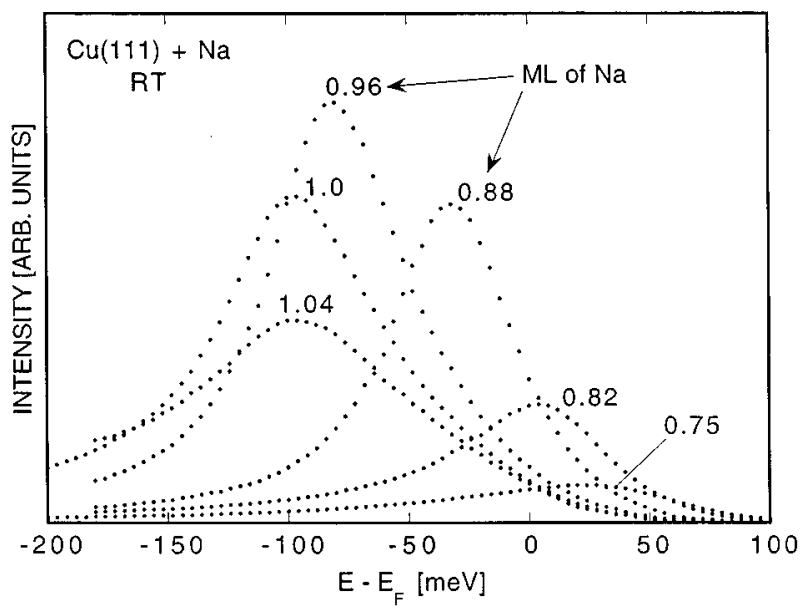

FIG. 1. Photoelectron energy spectra at initial energies near the Fermi level at a photon energy of $3.82 \mathrm{eV}$ recorded along the surface normal of $\mathrm{Cu}(111)$ covered by the different monolayer amounts of $\mathrm{Na}$ given in the diagram. The light is $p$ polarized and the incidence angle $79^{\circ}$.

saturation of the shift. Another measure of the adsorbed amount is given by the work-function change, which is obtained either from the width of the photoelectron energy spectrum or by the diode method using the monochromator of the loss spectrometer as beam source.

\section{RESULTS}

\section{A. Energy shifts}

Figure 1 shows spectra recorded along the surface normal of the $\mathrm{Cu}(111) / \mathrm{Na}$ sample at different $\mathrm{Na}$ coverages in the monolayer range with the sample kept at room temperature (RT). For coverages above $0.82 \mathrm{ML}$ a peak corresponding to states near the Fermi energy is observed. The peak shifts to lower initial energy as the coverage is increased until saturation is reached at an energy of $-97 \mathrm{meV}$. The initial state energy is given relative to $E_{F}$ and the minus sign indicates that the energy is below $E_{F}$. The zero of the energy scale is obtained from spectra recorded at low alkali-metal coverages when the monolayer state peak is not prominent and the spectra are rather flat near the Fermi edge. At coverages below $0.82 \mathrm{ML}$ the emission extending beyond the Fermi edge indicates that the Fermi cutoff function prevents clear observation of the state when it falls above $E_{F}$. That this is the case is demonstrated by the spectra obtained after compensation for the reduced occupancy of states above $E_{F}$ (Fig. 2). The spectra shown in Fig. 2 are thus the spectra shown in Fig. 1 multiplied by $1+e^{\left(E-E_{F}\right) / k T}$. With the high emission intensities obtained this procedure allows peaks to be resolved for energies below around $10 \mathrm{kT}$ above the Fermi level. Prior to multiplication a flat spectrometer background intensity is subtracted from the spectra. This has an appreciable effect only near the high-energy limit of the probed range. The Fermi-Dirac factor is calculated using the experimentally determined position and width of the Fermi edge at small Na coverages or high emission angles when the photoelectron energy spectrum is rather flat below the Fermi edge. ${ }^{5}$ Figure 3 shows a plot of the energy versus coverage

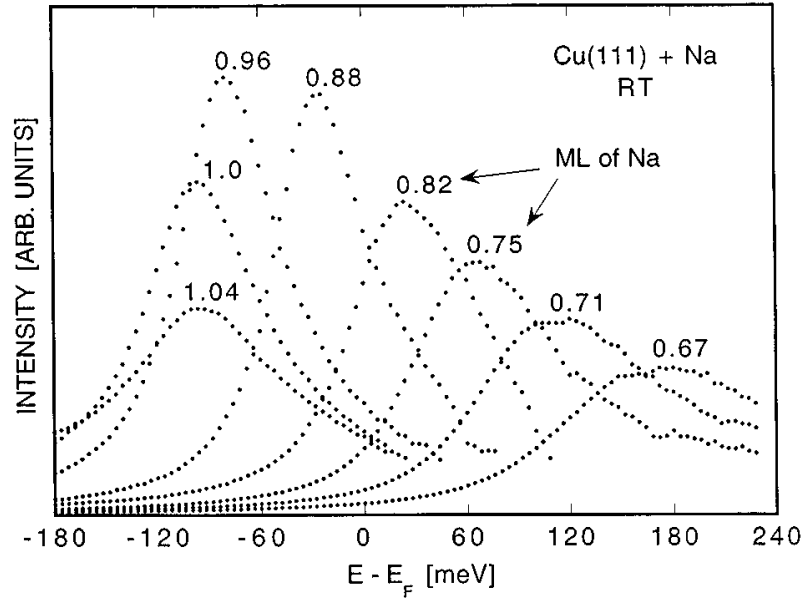

FIG. 2. The photoelectron energy spectra shown in Fig. 1 divided by the Fermi cutoff function.

for the peaks shown in Fig. 2. The center of peak corresponding to the point marked with parentheses in Fig. 3 has not been observed; only its low-energy tail, which has been fitted to a Lorentzian, has been observed (Fig. 4). As discussed below the low-energy side of the emission peaks has this line shape.

We thus observe an emission peak that shifts gradually to lower energy as the deposition time is increased and then, if a critical coverage is exceeded, remains at a nearly constant initial-state energy. The peak height passes a maximum at slightly lower coverage than needed to obtain saturation of the energy shift. The coverages given in the present paper are based on the assumption that the first monolayer is complete at saturation of the shift.

The width of the Fermi edge is used to determine the surface temperature. With an experimental resolution of around $5 \mathrm{meV}$ suggested by the $7-\mathrm{meV}$ width of the elastic beam transmitted by the loss spectrometer the main contribution to the width of the edge comes from the Fermi distribution at the temperatures used (above $128 \mathrm{~K}$ ). The width of the Fermi edge is measured either at low Na coverage or at large emission angles when the spectra are relatively flat near

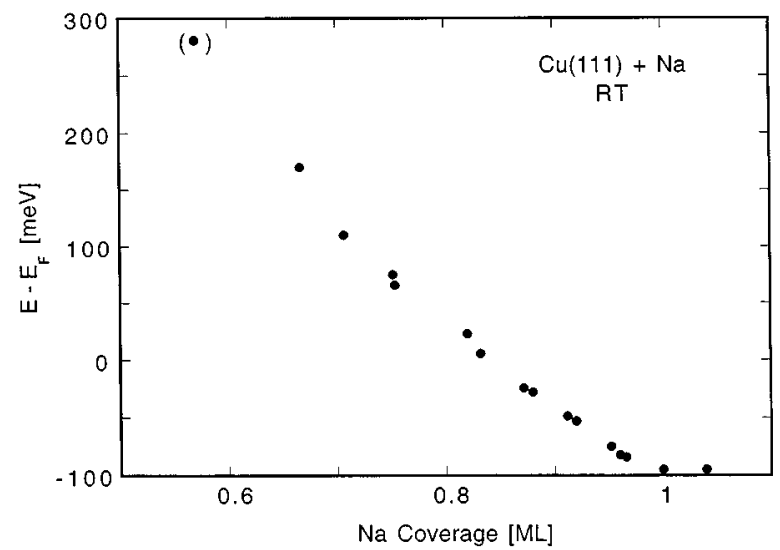

FIG. 3. Initial-state energy vs coverage for the emission peak (shown in Figs. 1 and 2) due to a discrete overlayer state characteristic of $\mathrm{Na}$ monolayers on $\mathrm{Cu}(111)$. 


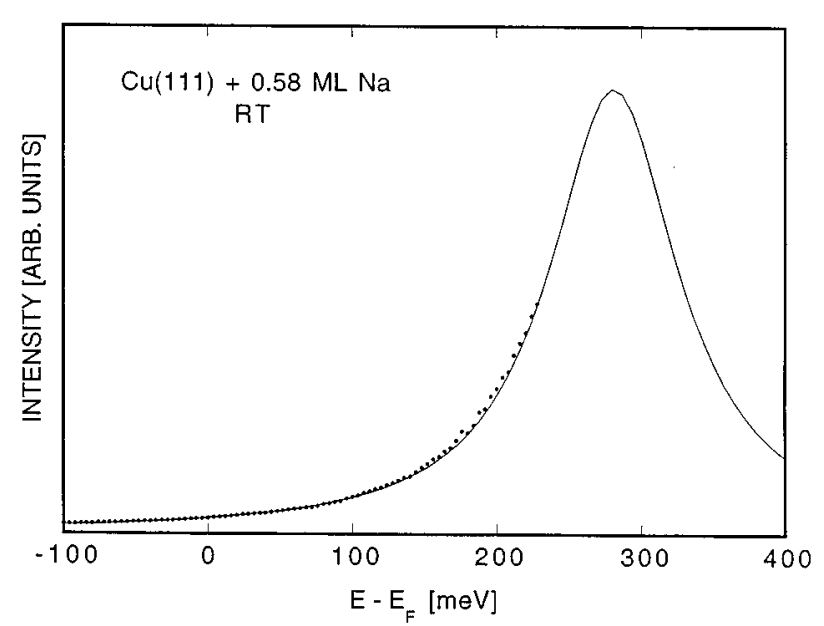

FIG. 4. The low-energy tail of an emission peak (filled circles) due to the discrete overlayer state characteristic of $\mathrm{Na}$ monolayers on $\mathrm{Cu}(111)$ at 0.58 -ML Na coverage fit to a Lorentzian (full drawn curve). The tail has been obtained by dividing the recorded spectrum with the Fermi cutoff function. The photon energy is $3.82 \mathrm{eV}$, the incidence angle $79^{\circ}$, and the spectrum is recorded along the surface normal.

the edge. As expected for a free-electron-like overlayer the energy has a minimum at $k_{\|}=0$ and is proportional to the square of the parallel wave vector (Fig. 5). Due to the low kinetic energy, around $1 \mathrm{eV}$, the trajectory of the photoelectrons and the effective mass value obtained, $0.5 m_{e}$, is sensitive to stray fields near the sample. The small range of $k_{\|}$ values in one direction (Fig. 5) is due to a limit set by an aperture admitting the light beam into the sample compartment.

When more than one atomic layer is adsorbed the emission intensity due to the monolayer state decreases and another peak, approximately $100 \mathrm{meV}$ above $E_{F}$, ascribed to two-atomic-layers-thick parts of the overlayer appears (Fig. 6). This state is observed for coverages above around 1.3 ML. The energy distributions shown in Fig. 6 are derived from spectra recorded with the sample kept at $165 \mathrm{~K}$ during deposition and measurement. The results obtained at RT are quite similar but the peaks are somewhat better resolved at

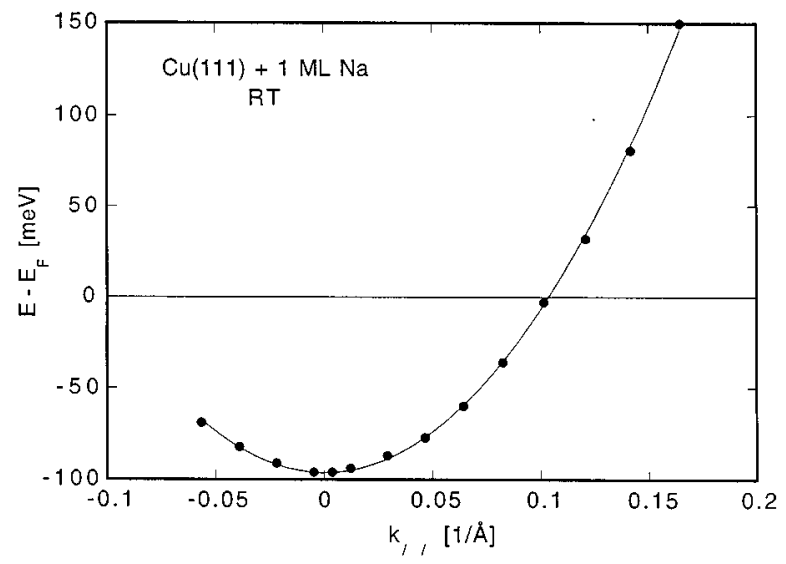

FIG. 5. Energy vs parallel wave vector for the discrete overlayer state characteristic of $\mathrm{Na}$ monolayers on $\mathrm{Cu}(111)$.

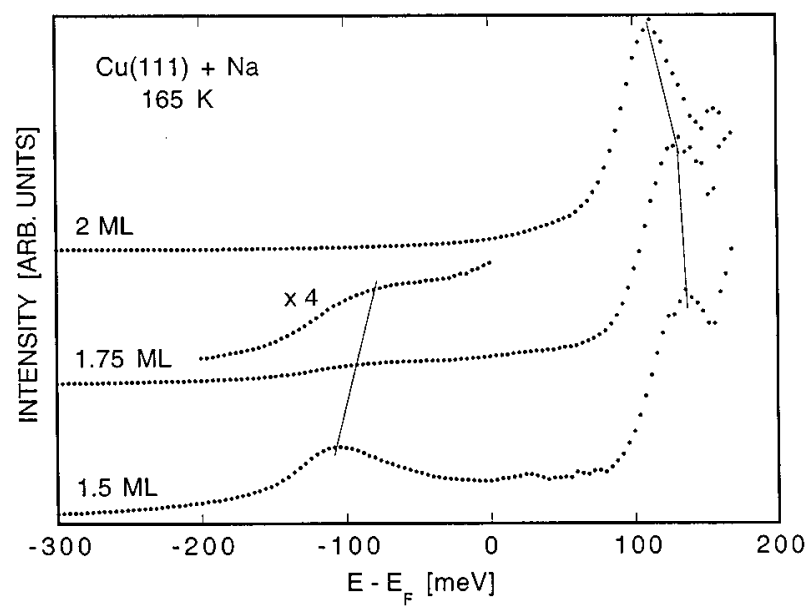

FIG. 6. Emission peaks obtained as in Fig. 2 for different $\mathrm{Na}$ coverages between 1 and $2 \mathrm{ML}$. The peaks at around $E-E_{F}$ $=-0.1 \mathrm{eV}$ and $0.1 \mathrm{eV}$ are due to one- and two-atomic-layers-thick areas of the $\mathrm{Na}$ film, respectively.

the lower temperature. The peak characteristic of two atomic layers shifts downwards by $25 \mathrm{meV}$ when the coverage is changed from 1.75 to $2 \mathrm{ML}$. The peak characteristic of oneatomic-layer-thick regions shows a similar shifts but to higher energy. The shifts observed during the formation of the second layer are thus small compared to the shift observed in the monolayer coverage range.

\section{B. Widths and line shapes}

The widths of the emission peaks depend on the coverage and on the temperature. The coverage dependence is particularly strong in the monolayer range (Fig. 7). The width is small and almost constant between 0.87 and $0.96 \mathrm{ML}$ of $\mathrm{Na}$ and rises sharply above $0.96 \mathrm{ML}$. Also the line shape depends on coverage. At coverages that produce a narrow emission peak the line shape is quite well reproduced by a Lorentzian (Fig. 8), while the peak is asymmetric for coverages giving a broader peak. Even then the low-kinetic-energy side of the peak is Lorentzian and narrow compared to the high-energy side.

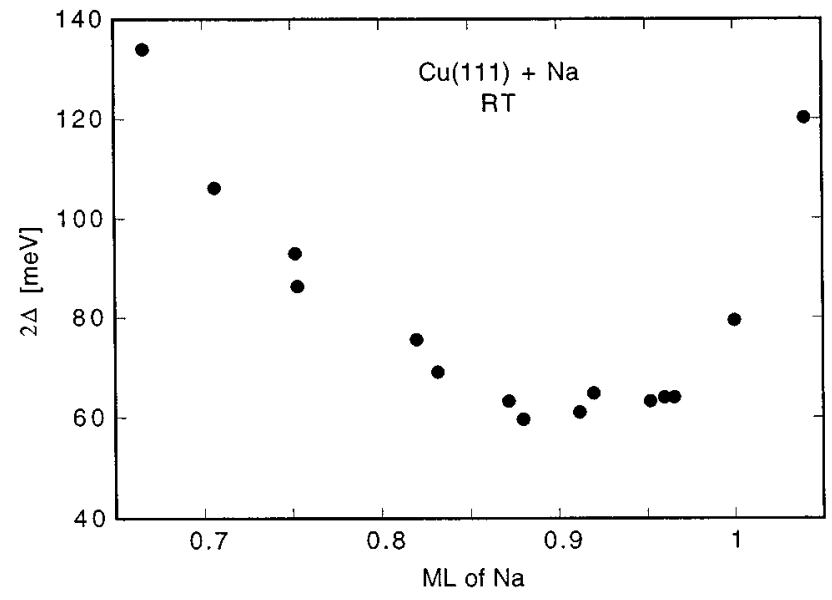

FIG. 7. The width parameter $2 \Delta$ vs coverage for the monolayer state emission peak measured at RT. 


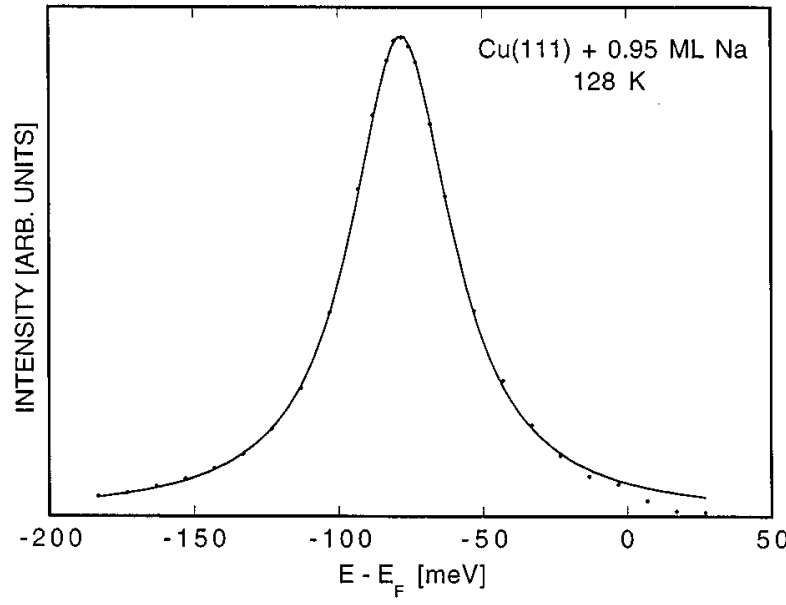

FIG. 8. A Lorentzian with a full width at half maximum $2 \Delta$ of 42-meV (full drawn curve) fit to the emission peak (dots) recorded at $130 \mathrm{~K}$ for $0.95 \mathrm{ML}$ of $\mathrm{Na}$ on $\mathrm{Cu}(111)$ along the surface normal at 3.82-eV photon energy.

The temperature dependence of the width was studied mainly at coverages near one full atomic layer when the peaks are narrow. As shown in Fig. 9 the width changes nearly linearly with temperature in the probed range between $128 \mathrm{~K}$ and RT. The full drawn curve in Fig. 9 is obtained, as discussed further below, by fitting a theoretical expression for the width to the experimental values. Also for a twoatomic-layer-thick film there is, as shown in Fig. 10, a substantial narrowing of the linewidth as the temperature is reduced. Since the 2-ML state lies $0.1 \mathrm{eV}$ above $E_{F}$ the occupancy is strongly reduced by cooling. The spectrum therefore changes dramatically as the temperature is reduced (Fig. 10). One should note that the intensity is reduced not only above the Fermi edge but also below it. The shoulder above $E_{F}$ (Fig. 10) is strongly reduced due to the lower population of the state. For energies below $E_{F}$ the Fermi

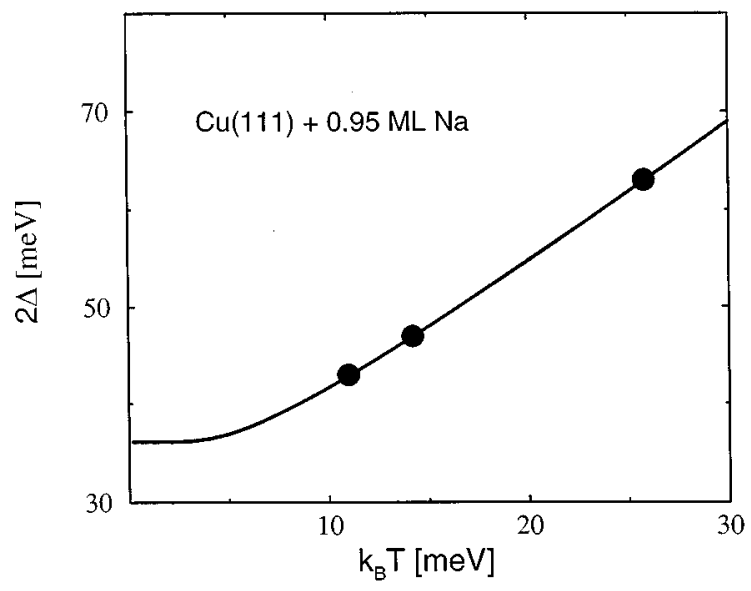

FIG. 9. The width parameter $2 \Delta$ plotted vs temperature for monolayer state emission peaks measured at 0.95-ML Na coverage on $\mathrm{Cu}(111)$. The solid line is a fit to the experimental points (filled circles) obtained, as discussed in the text, from Eq. (4) with adjustable parameters $\Delta_{0}$ and $\lambda$ to account for contributions to the width due to impurities and defects and due to the electron-phonon coupling, respectively.

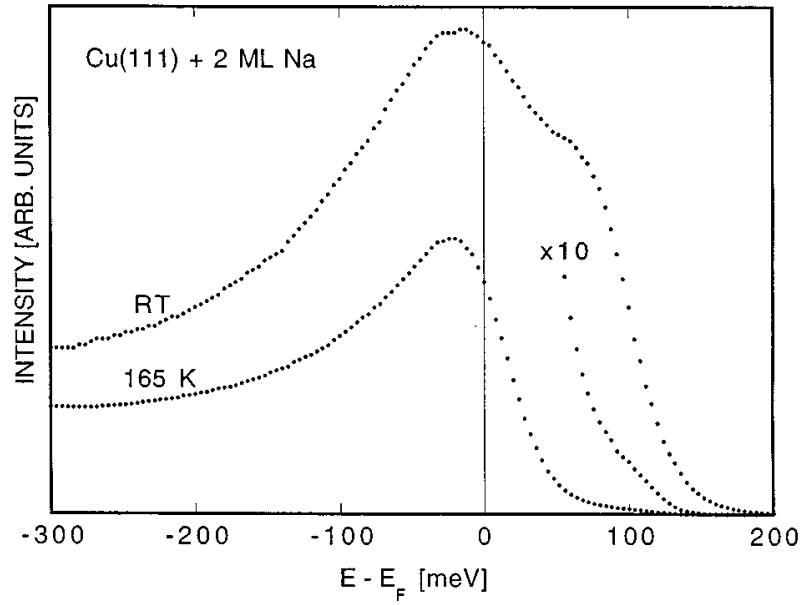

FIG. 10. Photoelectron energy spectra recorded at 3.82-eV photon energy along the surface normal of $\mathrm{Cu}(111)$ covered with $2 \mathrm{ML}$ of $\mathrm{Na}$ with the sample at RT (upper spectrum) and at $165 \mathrm{~K}$.

factor increases but the higher emission intensity expected from this is more than compensated for by the narrowing of the emission line as the temperature is reduced. We also note that the intensity is reduced as the temperature is lowered (Fig. 11). Since the peak height obtained after compensating for the Fermi factor is quite sensitive to errors in the temperature determination for a state this far above the Fermi level we will not base any discussion on the temperature dependence of the intensity. The two other parameters of interest, the energy and the linewidth, are much less affected by uncertainties in the temperature determination.

\section{DISCUSSION}

\section{A. Energy shifts}

While the discrete states studied here are found in adsorbed Na films similar states would be expected to appear if the films could be prepared surrounded by vacuum. This is shown by band-structure calculations, which have been made for 1-5 ML of $\mathrm{Li}$ (Ref. 6) and for a $\mathrm{Na}$ monolayer in

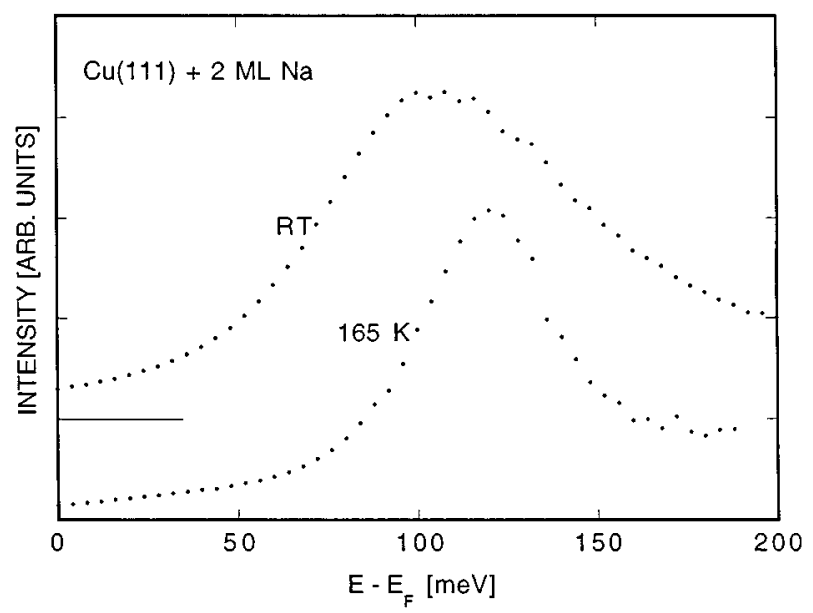

FIG. 11. The photoelectron energy spectra shown in Fig, 10 divided by the Fermi cutoff function. 
vacuum. ${ }^{7}$ For the Na monolayer and the next lowest band the energy minimum at $k_{\|}=0$ is calculated to lie $0.5 \mathrm{eV}$ above $E_{F}$. If the monolayer is placed on a $\mathrm{Cu}(111)$ substrate the energy falls within the $L$ gap of the $s, p$ band, which means that one of the vacuum tails becomes replaced by an oscillatory tail into the substrate. The phase shifts are different at the vacuum barrier and the metal-metal interface and therefore the energy is different when the layer is adsorbed. ${ }^{9}$ The energy is downshifted to $0.1 \mathrm{eV}$ below $E_{F}$ and the band is occupied over a small area at the center of the surface Brillouin zone as observed here (Fig. 5). As shown by the calculations for Li a $k_{\|}=0$ state appears close above $E_{F}$ also if the film is two atomic layers thick and the corresponding $\mathrm{Na}$ state is observed here $0.1 \mathrm{eV}$ above the Fermi level. For energies lower than this the calculations show that there are two more states at $k_{\|}=0$. These fall below the $L$ gap and, upon adsorption, form resonances with the substrate $s, p$-band electrons. The energies are below the range probed with the small photon energy used here. With photon energies around $6 \mathrm{eV}$ the resonance with the highest energy was recently observed. ${ }^{8}$

Quantum-well states near below $E_{F}$ for alkali-metal monolayers on $\mathrm{Cu}(111)$ have been observed previously ${ }^{9-13}$ but not the gradual shift in energy observed here with increasing coverage. However, a shift of approximately the magnitude observed has been predicted using a simple phase-shift analysis. ${ }^{2}$ In qualitative terms the downward shift is produced by a more attractive potential in the overlayer as more $\mathrm{Na}$ atoms are accommodated. This shift is balanced only in part by a shift to higher energy expected due to the increase of the work function typical of alkali-metal monolayers when the coverage is increased from half to full monolayer coverage. For $\mathrm{Na}$ on $\mathrm{Cu}(111)$ the work function increases from a minimum value of $2.1 \mathrm{eV}$ to $2.7 \mathrm{eV}$ in this coverage range.

For quantitative estimates the quantum-well state energy is obtained from the phase condition $\Phi_{B}+\Phi_{C}+2 \Phi_{D}$ $=2 \pi m, m=0,1,2, \ldots$, where $\Phi_{B}$ and $\Phi_{C}$ are the phase shifts at the vacuum barrier and at the overlayer-substrate interface respectively and $\Phi_{D}$ is the phase accumulation across the overlayer. The application of this phase model to various overlayer-substrate combinations has been discussed in recent reports. ${ }^{14,15}$ For Na overlayers we assume the potential to be flat in the overlayer, which means that $\Phi_{D}=2 \mathrm{kNL}$, where $L$ is the thickness of an atomic layer and $N$ the number of layers. For the states $0.1 \mathrm{eV}$ below and above $E_{F}$ for 1 and 2 ML, respectively, the phase condition is satisfied for $m=N$. To obtain the wave vector $k$ in the submonolayer range it is assumed that the monolayer defines a volume that is increasingly filled with uniformly distributed atoms as the coverage increases. Provided the overlayer is neutral one then expects the Fermi energy to be proportional to $\Theta^{2 / 3}$, where $\Theta$ is the coverage in units such that $\Theta=1$ is one full monolayer. To fix the proportionality constant we furthermore assume that the electron density at full monolayer coverage is the same as for bulk Na. To estimate the influence of the work-function change on the energy we have used the approximation $^{16} \Phi_{B} / \pi=\left[(3.4 \mathrm{eV}) /\left(E_{v}-E\right)\right]^{1 / 2}-1$, where $E_{V}$ is the vacuum level. With $L=3.05 \AA$, which is the thickness of a close-packed $\mathrm{Na}$ layer, and using the measured

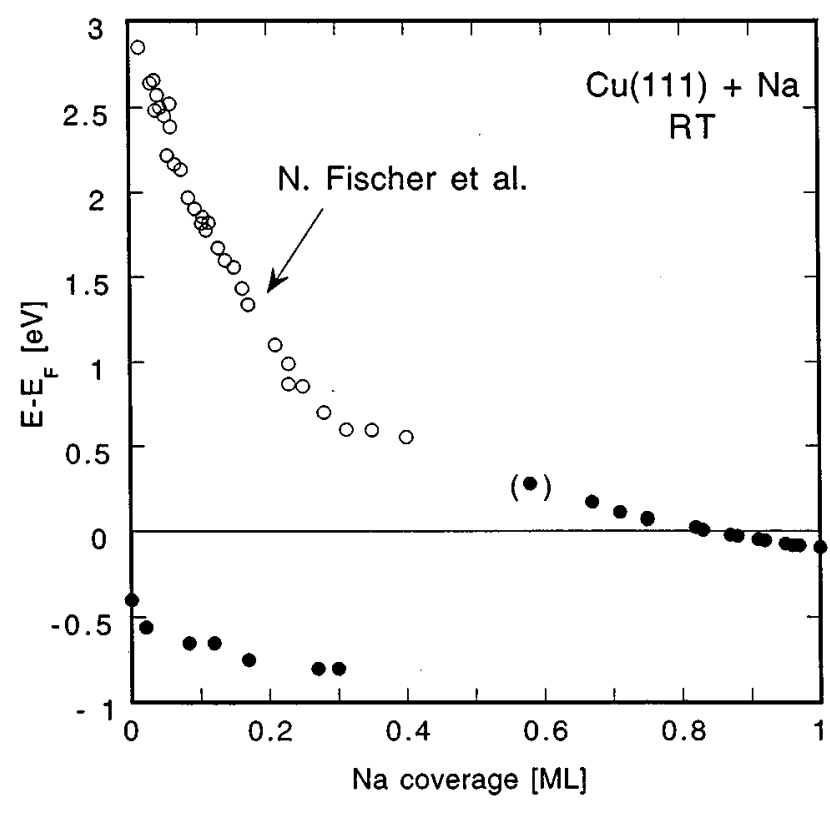

FIG. 12. Energy vs $\mathrm{Na}$ coverage on $\mathrm{Cu}(111)$ for filled states observed here (filled circles) and empty states observed by Fischer et al. (Ref. 3) (open circles). The point within parentheses is obtained as shown in Fig. 4.

coverage dependence of the work function we obtain a shift with the correct sign and a magnitude which is $65 \%$ of the measured. ${ }^{2}$

From the emission angle dependence of the spectrum (Fig. 5) it is clear that the overlayer state observed in normal emission is the low-energy edge of an energy band. This band becomes partially occupied as the coverage exceeds $0.85 \mathrm{ML}$ when the peak shifts below the Fermi level. On this point our results are in apparent conflict with previous work, ${ }^{3,10}$ which suggests that there is a stepwise change of the electronic structure in this coverage range producing a stepwise change in the occupancy of the subband. A quite similar abrupt change was observed also for $\mathrm{Li}$ on $\mathrm{Be}(0001){ }^{4}$ Based on this observation and the fact that, as for $\mathrm{Na}$ on $\mathrm{Cu}(111)$, there is a disorder-order structural transformation in the coverage range of interest it was suggested that the stepwise change could be a manifestation of a transformation to metal character for the overlayer.

We note that our measured and estimated energies approach the empty state energies found by Fischer et al. ${ }^{3}$ If our photoemission results are combined with the PPES results by Fischer $e t a l .^{3}$ one finds that one state can be monitored over almost the entire monolayer coverage range, from low coverages when the energy is about $1 \mathrm{eV}$ below the vacuum level to full monolayer coverage when it is $0.1 \mathrm{eV}$ below the Fermi level (Fig. 12). One notes in Fig. 12 that there is still a gap in coverage and energy between the two experiments, which, however, may be explained by the energy limits of the experimental methods used. Simply by changing the monolayer coverage one may thus modify the quantum well such that a state may shift from the image potential region to the occupied range of energies.

A question that remains to be answered is how far towards the low coverage limit this range of continuously tunable quantum-well-state energy extends. According to the 
PPES results ${ }^{3}$ there is such a limit and the shifting state was described as an alkali-adsorption-induced state. On the other hand no similar limit is observed for the shift of the $\mathrm{Cu}(111)$ surface state $0.4 \mathrm{eV}$ below the Fermi level. Regarding the low coverage limit one should also point out that, due to surface defects, the results obtained for small adsorbate densities may not be representative of terrace sites. From corelevel spectra for alkali metals on A1(111) it was concluded that initially upon deposition the adatoms assume defect sites. ${ }^{17}$

The fact that we observe two quantum-well-state emission peaks for a wide range of coverages between one and two atomic layers shows that the second layer grows via the formation of monolayer high islands on top of the first atomic layer. The small downshift, by around $25 \mathrm{meV}$, observed for the state as the coverage is varied is ascribed to a larger average size of the islands when more $\mathrm{Na}$ is adsorbed. The island size places a limit on how small lateral wave vectors that can be used to form the quantum-well state. Since, according to the dispersion shown above (Fig. 5), the energy is proportional to the square of the lateral wave vector the observed shift is in the direction expected from the lateral quantum size effect.

\section{B. Width and line shape}

As may be expected the widths of the emission peaks reflect the disorder of the overlayer. According to lowenergy electron diffraction observations ${ }^{18}$ the $\mathrm{Na}$ atoms form an ordered structure for coverages above $0.89 \mathrm{ML}$ and in the range between 0.87 and $0.96 \mathrm{ML}$ the peak width is small (Fig. 7). The probable explanation for the increased width measured when the coverage is increased beyond $0.96 \mathrm{ML}$ (Fig. 7) is that $\mathrm{Na}$ atoms start to occupy sites in the second layer already before the first layer is complete. This makes the monolayer quantum well less well defined and the energy spread bigger. When the emission peak is narrow the line shape is close to a Lorentzian (Fig. 8). As the coverage is decreased below $0.87 \mathrm{ML}$ the increased width is accompanied by an increased asymmetry, with a lower slope on the high-energy side. The low-energy part of the peak is still close to a Lorentzian.

There are few previous observations of line shapes and widths at different temperatures for discrete valence electron states close to $E_{F}$. Recently results have been reported for emission lines due to surface states near below $E_{F}$ in the $s, p$ band gap at $L$ characteristic of the (111) surface of the noble metals. ${ }^{19,20}$ As found here for overlayer states the emission out of these surface states is characterized by a Lorentzian line shape. The width measured for the $\mathrm{Cu}(111)$ surface state emission peak is found in Ref. 20 to increase linearly with temperature in the temperature range studied, $30-600 \mathrm{~K}$. The line shape, the absolute values observed for the width and its temperature dependence, suggests that the width can be ascribed to a lifetime that is limited by electronphonon scattering. As far as we know there is no theoretical prediction for valence levels regarding photoemission widths and line shapes due to thermal motion. More typical for valence states is that the initial energy is far enough from $E_{F}$ that electron-electron scattering limits the hole lifetime. According to Quinn the energy spread at $T=0$ due to the lim- ited hole lifetime for a free-electron-like metal is to a good approximation $^{21,22}$ given by $\operatorname{Im} \Sigma=0.001 r_{S}^{5 / 2}\left(E-E_{F}\right)^{2} \mathrm{eV}$. This theoretical estimate is of the right order to account for the width, $0.44 \mathrm{eV}$ full width at half maximum, observed for a surface state on $\mathrm{Be}(0001) 2.8 \mathrm{eV}$ below $E_{F}$ and the emission peak has the expected Lorentzian shape. ${ }^{23}$ In the present case, with electron energies within $0.1 \mathrm{eV}$ of the Fermi level and an $r_{S}$ value somewhere between those for $\mathrm{Cu}$ and $\mathrm{Na}$, the estimated width is less than $1 \mathrm{meV}$. At nonzero temperature there is also an additional term, proportional to $T^{2}$, which is expected to contribute less than $1 \mathrm{meV}$ at temperatures of interest. $^{24}$

A straight line fitted to the widths plotted versus temperature in Fig. 9 has a slope of $0.12 \mathrm{meV} / \mathrm{K}$ for 0.95-ML Na. In the recent work on $\mathrm{Cu}(111)$ a similar linear relationship was found for the photo-emission width of the surface state 0.4 $\mathrm{eV}$ below $E_{F}$ (Ref. 20) and the authors note that the slope obtained, $0.074 \mathrm{meV} / \mathrm{K}$, gives $\lambda=0.14$ for the electronphonon coupling constant. This value is close to the theoretically estimated value obtained for bulk $\mathrm{Cu}$ when averaging over the Fermi surface, $\lambda=0.15$. $^{25}$

Analyzing the theoretically derived formula for the phonon broadening, presented by Grimval, ${ }^{25}$ McDougall et al. ${ }^{20}$ discuss the temperature and binding energy dependence of the width. They point out that the expression for the phonon broadening, $2 \Delta=2 \pi \lambda k_{B} T$, valid for thermal energies well above the phonon energies, ${ }^{26}$ should be approximately valid also for thermal energies well below the maximum phonon energy. Furthermore they indicate that the width is nearly independent of the binding energy in the temperature range from zero to RT, as long as the binding energy exceeds the phonon energies.

In the present case the monolayer state is a hybrid with the electron spending approximately half of its time in the $\mathrm{Cu}$ substrate and half in the $\mathrm{Na}$ overlayer. For a $\mathrm{Na}$ coverage of $0.95 \mathrm{ML}$ the slope obtained when the measured width is plotted versus $k_{B} T$ (Fig. 9) yields $\lambda=0.24$. This could then reflect a larger coupling constant for $\mathrm{Na}$ than for $\mathrm{Cu}$. A slightly stronger temperature dependence is observed for the width of the 2-ML state and this could then be explained by the fact that this electron spends a larger fraction of its time in the alkali metal. However, the theoretically estimated $\lambda$ values for $\mathrm{Na}(0.16 \pm 0.03)$ and for $\mathrm{Cu}(0.15 \pm 0.03)$ (Ref. $25)$ are nearly equal and both smaller than the value obtained in the present experiment. This suggests that either the electron-phonon coupling is stronger in ultrathin $\mathrm{Na}$ films than in bulk $\mathrm{Na}$ or that there are additional temperaturedependent broadening mechanisms not accounted for.

From a theoretical analysis of the self-energy of the hole state we have derived an expression for the phonon-induced lifetime broadening. ${ }^{27}$ The overlayer electron states $\Psi_{m, k_{\|}}$ are quantized $(m)$ in the direction normal to the surface and are of plane-wave type parallel to it. According to this model $^{27}$ the main contribution to the width is due to intersubband scattering, which implies that the electrons couple predominantly to phonons polarized perpendicular to the surface. The phonon modes of particular interest are therefore the organ-pipe-like ones, which have been studied extensively for alkali-metal overlayers using inelastic $\mathrm{He}$ scattering. ${ }^{28-30}$ 


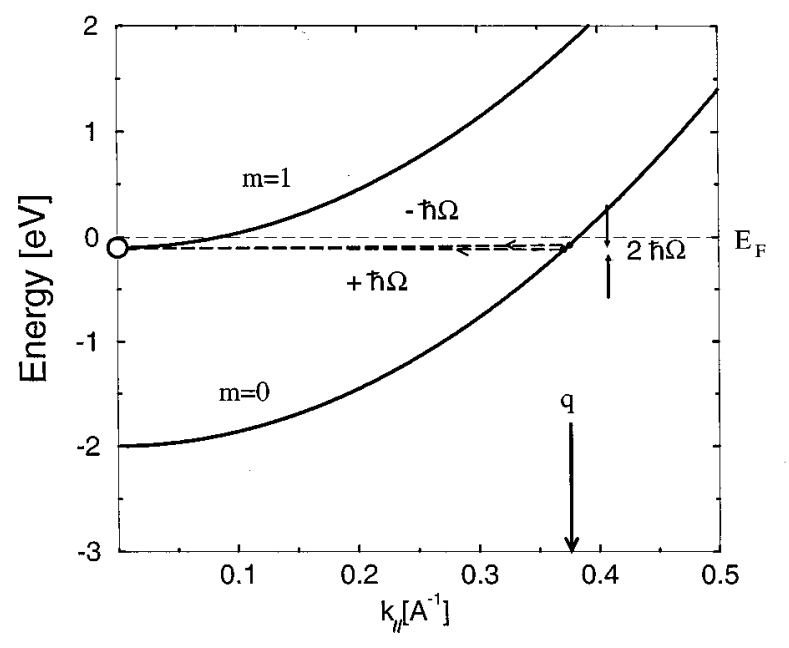

FIG. 13. A schematic subband diagram where the thick dashed lines indicate electron scattering involving phonon absorption $(+\hbar \Omega)$ and emission $(-\hbar \Omega)$. The hole, corresponding to $0.95 \mathrm{ML}$ of $\mathrm{Na}$ on $\mathrm{Cu}(111)$, is indicated by the open circle $80 \mathrm{meV}$ below the Fermi level $\left(E_{F}\right)$. The vertical arrow indicates the magnitude of the phonon wave vector. The lower subband is obtained by assuming a free electron band structure for the Na monolayer.

In Fig. 13 the intersubband scattering from subband $m$ $=0$ to the hole state at $\Gamma$ in subband $m=1$ is indicated. This subband picture is a simplification since, for the real system, the $m=0$ subband falls in the energy range of the substrate $s, p$ band and therefore it is resonance broadened. The two dashed lines correspond to absorption $(+\hbar \Omega)$ and emission $(-\hbar \Omega)$ of a phonon. The width, given by the imaginary part of the self-energy, can be written as ${ }^{27}$

$$
2 \Delta=(\pi / 2) \lambda \hbar \Omega g(T),
$$

where $\lambda$ is the electron-phonon coupling constant, and $\hbar \Omega$ the energy of the characteristic phonon mode. The temperature dependence is described by the function $g(T)$,

$$
\begin{aligned}
g(T)= & 2 \operatorname{coth}\left(\hbar \Omega / 2 k_{B} T\right)+\tanh \left[\left(E-E_{F}-\hbar \Omega\right) / 2 k_{B} T\right] \\
& -\tanh \left[\left(E-E_{F}+\hbar \Omega\right) / 2 k_{B} T\right],
\end{aligned}
$$

where $E$ is the hole state energy and $E_{F}$ the Fermi energy. In the high-temperature limit one obtains $2 \Delta=2 \pi \lambda k_{B} T$. In the model $\lambda$ has the form

$$
\lambda=\left|\left\langle\frac{d u_{0}}{d z} \mid u_{1}\right\rangle+\left\langle u_{0} \mid \frac{d u_{1}}{d z}\right\rangle\right|^{2} \frac{1}{n_{a}} \frac{m_{e}}{M}\left(\frac{V_{q}}{\hbar \Omega}\right)^{2},
$$

where $u_{m}(z)$ is the electron wave function normal to the surface, $n_{a}$ the density of adsorbed atoms, $m_{e}$ the electron mass, $M$ the mass of the adsorbed atom, $V_{q}$ a Fourier component of the electron potential where $q$ is the parallel wave vector of the phonon mode with angular frequency $\Omega$.

In order to compare with the experimentally determined widths we add a term $2 \Delta_{0}$ to account for presumably temperature independent contributions to the width due to impurities and defects. The expression used for comparison with experiment therefore reads

$$
2 \Delta=2 \Delta_{0}+(\pi / 2) \lambda \hbar \Omega g(T) .
$$

In Fig. 9 we display the theoretical width obtained from this formula when the parameters $\Delta_{0}$ and $\lambda$ are used to fit the experimental widths shown in the same diagram. For the case of $0.95 \mathrm{ML} \mathrm{Na}$ on $\mathrm{Cu}(111)$ we have $E-E_{F}=$ $-80 \mathrm{meV}$ and $\hbar \Omega=18 \mathrm{meV}^{28}$

Thus $\left|E_{F}-E\right| \gg \hbar \Omega$, which according to the expression above results in the asymptotic behavior, $2 \Delta \rightarrow 2 \Delta_{0}$ $+\pi \lambda \hbar \Omega$ as $T \rightarrow 0$. The fit to the experiment gives $2 \Delta_{0}$ $=23 \mathrm{meV}$ and as before $\lambda=0.24$. From the comparison between experiment and theory we are thus able to estimate the influence of impurities and defects on the measured linewidths. It is furthermore interesting to note that, according to Eq. (4), at low temperatures $\left(k_{B} T<\hbar \Omega\right)$ the temperature dependence of the width depends strongly on the hole energy, $E$, when $E$ is varied in the range $\left|E-E_{F}\right|<\hbar \Omega$. This will be discussed elsewhere. ${ }^{27}$

The asymmetric peak shape observed in the low coverage range of the experiment is ascribed to disorder in the monolayer. Photoelectrons heading in other directions may be scattered by the atoms in the overlayer into the spectrometer. The asymmetry is then explained by the positive dispersion of the energy band, which means that quasielastic scattering events will contribute only to the high-energy side of the emission peak.

\section{CONCLUSIONS}

High-resolution photoemission spectroscopy has been used to study quantum-well-type states characteristic of one and two atomic layers of $\mathrm{Na}$ on $\mathrm{Cu}(111)$. The $k_{\|}=0$ state characteristic of a monolayer shifts gradually to lower energies as the coverage is increased. Above $0.85 \mathrm{ML}$ the state is below the Fermi energy and when full monolayer coverage is exceeded, it remains at a nearly constant energy, $0.1 \mathrm{eV}$ beneath $E_{F}$. The peak height reaches a maximum at a slightly lower coverage than needed for saturation of the energy shift. Combining the present and previous results one finds that surface and overlayer state energies shift in a gradual manner as the monolayer coverage is changed. If the coverage is increased above $1 \mathrm{ML}$ the intensity of this peak decreases and from approximately $1.3 \mathrm{ML}$ a state characteristic of 2 ML is observed. Between 1.7 and $2 \mathrm{ML}$ the 2-ML state shows a downshift by $25 \mathrm{meV}$ to $0.1 \mathrm{eV}$ above $E_{F}$. The shift is ascribed to lateral quantization due to the limited size of islands formed by $\mathrm{Na}$ atoms in the second atomic layer on top of the first full layer.

The widths of the emission peaks depend on the temperature and the coverage. At nearly full monolayer coverage the quantum-well state gives a narrow Lorentzian line having a width that increases linearly with temperature in the range probed (130-295 K). The width is assigned to a phonon limited lifetime, which is a factor of around 2 shorter than expected for a state near $E_{F}$ in bulk $\mathrm{Na}$ or $\mathrm{Cu}$. For the less well ordered monolayers the line shape for electrons emitted along the surface normal is asymmetric with a Lorentzian line shape only on the low-energy side. The asymmetry is ascribed to quasielastic scattering, which contributes to the spectrum only on the high-energy side of the emission peak.

\section{ACKNOWLEDGMENTS}

This work has been supported by grants from the Swedish Natural Science Research Council and the Knut and Alice Wallenberg Foundation. 
1 A. M. Bradshaw, H. P. Bonzel, and G. Ertl, Physics and Chemistry of Alkali Metal Adsorption (Elsevier, Amsterdam, 1989).

${ }^{2}$ S. A. Lindgren and L. Walldén, Phys. Rev. B 38, 3060 (1988).

${ }^{3}$ N. Fischer, S. Schuppler, T. Fauster, and W. Steinmann, Surf. Sci. 314, 89 (1994).

${ }^{4}$ G. M. Watson, P. A. Brühwiler, H. J. Sagner, K. H. Frank, and E. W. Plummer, Phys. Rev. B 50, 17678 (1994).

${ }^{5}$ A. Carlsson, S. A. Lindgren, C. Svensson, and L. Walldén, Phys. Rev. B 50, 8926 (1994).

${ }^{6}$ J. C. Boettger and S. B. Trickey, Phys. Rev. B 45, 1363 (1992).

${ }^{7}$ E. Wimmer, J. Phys. F 13, 2313 (1983).

${ }^{8}$ A. Carlsson, D. Claesson, S.-Å. Lindgren, and L. Walldén, Phys. Rev. Lett. 77, 346 (1996).

${ }^{9}$ S. Å. Lindgren and L. Walldén, Phys. Rev. Lett. 59, 3003 (1987).

${ }^{10}$ S. A. Lindgren and L. Walldén, Solid State Commun. 34, 671 (1980).

${ }^{11}$ N. Fischer, S. Schuppler, R. Fischer, Th. Fauster, and W. Steinmann, Phys. Rev. B 43, 14722 (1991).

${ }^{12}$ N. Fischer, S. Schuppler, R. Fischer, Th. Fauster, and W. Steinmann, Phys. Rev. B 47, 4705 (1993).

${ }^{13}$ R. Dudde and B. Reihl, Surf. Sci. 287/288, 614 (1993).

${ }^{14}$ A. Beckman, M. Klaua, and K. Meinel, Phys. Rev. B 48, 1844 (1993).

${ }^{15}$ N. V. Smith, N. B. Brookes, Y. Chang, and P. D. Johnson, Phys. Rev. B 49, 332 (1994).
${ }^{16}$ E. G. McRae and M. L. Kane, Surf. Sci. 108, 435 (1981).

${ }^{17}$ J. N. Andersen, E. Lundgren, R. Nyholm, and M. Qvarford, Surf. Sci. 289, 307 (1993).

${ }^{18}$ D. Tang, D. McIlroy, X. Shi, and D. Heskett, Surf. Sci. Lett. 255, L497 (1991).

${ }^{19}$ R. Paniago, R. Matzdorf, G. Meister, and A. Goldmann, Surf. Sci. 336, 113 (1995).

${ }^{20}$ B. A. McDougall, T. Balasubramanian, and E. Jensen, Phys. Rev. B 51, 13891 (1995).

${ }^{21}$ J. J. Quinn and R. A. Ferrell, Phys. Rev. 112, 812 (1958).

${ }^{22}$ J. J. Quinn, Phys. Rev. 126, 1453 (1962).

${ }^{23}$ R. A. Bartynski, E. Jensen, T. Gustafsson, and E. W. Plummer, Phys. Rev. B 32, 1921 (1985).

${ }^{24}$ D. Pines and P. Noziere, The Theory of Quantum Liquids (Benjamin, New York, 1969).

${ }^{25} \mathrm{G}$. Grimvall, The Electron-Phonon Interaction in Metals (NorthHolland, New York, 1981).

${ }^{26}$ M. J. G. Lee, Phys. Rev. B 2, 250 (1970).

${ }^{27}$ B. Hellsing, S.-A. Lindgren, and L. Walldén (unpublished).

${ }^{28}$ G. Benedek, J. Ellis, A. Reichmuth, P. Ruggerone, H. Schief, and J. P. Toennies, Phys. Rev. Lett. 69, 2951 (1992).

${ }^{29}$ N. S. Ruo, P. Ruggerone, and J. P. Toennies, Phys. Rev. B 54, 5051 (1996).

${ }^{30}$ E. Hulpke, J. Lower, and A. Reichmuth, Phys. Rev. B 53, 13901 (1996). 